\title{
Strategies for a successful hepatic artery anastomosis in liver transplantation: A review of 51 cases
}

\author{
Bien-Keem Tan ${ }^{1}{ }_{F R C S}$, Hui Chai Fong ${ }^{1}$ MRCS, Ek-Khoon $\underline{\operatorname{Tan}}^{2}{ }_{F R C S}$, Jeyaraj Prema $\underline{\text { Raj }}{ }^{2,3}{ }_{F R C S}$
}

\begin{abstract}
Introduction: Hepatic artery reconstruction is a critical aspect of liver transplantation. The microsurgeon faces several challenges when reconstructing the hepatic artery-the donor hepatic artery stalk is short and often a poor match for the usually hypertrophic recipient vessels. Previous inflammation impedes vessel dissection, and recipient vessels have a tendency to delaminate with manipulation. We review 51 consecutive liver transplantations to highlight these problems and propose strategies for a successful reconstruction of the hepatic artery.

Methods: A prospective study involving all adult patients undergoing liver transplantation at the Singapore General Hospital from January 2015 to December 2018 was undertaken. All hepatic artery anastomoses were performed by 2 microsurgeons at 10x magnification. Patients were started on a standard immunosuppressive regimen. Postoperative ultrasound scans on days 1, 3, 5, 7, 9 and 14 were used to confirm arterial patency.

Results: There were 51 patients who underwent liver transplantation during the study period. Of this number, 31 patients received deceased donor grafts and 20 received living donor grafts. A total of 61 anastomoses were performed ( 5 dual anastomosis, 4 radial artery interposition grafts) with 1 case of hepatic artery thrombosis that was successfully salvaged. The mean (range) postoperative resistive index and hepatic artery peak systolic velocity were $0.69(0.68-0.69)$ and $1.0 \mathrm{~m} / \mathrm{s}(0.88-1.10 \mathrm{~m} / \mathrm{s})$, respectively.

Conclusion: Hepatic artery thrombosis after liver transplantation is poorly tolerated. The challenges of hepatic artery reconstruction in liver transplantation are related to vessel quality and length. The use of microsurgical technique, appropriate recipient vessel selection, minimisation of vessel manipulation with modified instruments, variation in anastomosis techniques, and use of radial artery interpositional grafts are useful strategies to maximise the chances of success.
\end{abstract}

Ann Acad Med Singap 2021;50:679-85

Keywords: Hepatic artery, hepatology, liver transplant, microsurgery, plastic surgery

\section{INTRODUCTION}

Liver transplantation is a life-saving procedure for patients with chronic end-stage liver disease, and selected patients with acute liver failure. While $75 \%$ of total blood flow into the liver comes from the portal vein, the sole supply of oxygenated blood to the biliary system comes from the hepatic artery. ${ }^{1}$ A patent hepatic arterial anastomosis is critical in liver transplantation as thrombosis can result in ischaemia of the bile duct, presenting as biliary leak and resulting in eventual liver failure. Hepatic artery thrombosis leads to retransplantation in $50-75 \%$ of patients, with a mortality rate approaching $50 \%{ }^{2}$
While the introduction of microsurgical techniques has been instrumental in decreasing the rate of hepatic artery thrombosis, the microsurgeon faces unique challenges when reconstructing the hepatic artery. Firstly, the donor hepatic artery stalk is short, and of small calibre, mismatching with the recipient vessels. Secondly, previous peritonitis may render the coeliac axis fibrotic, impeding vessel dissection. Thirdly, a high proportion of patients undergoing liver transplantation have liver steatosis and atherosclerosis, or vessels that have been subjected to transarterial chemoembolisation (TACE),

\footnotetext{
${ }^{1}$ Department of Plastic, Reconstructive and Aesthetic Surgery, Singapore General Hospital, Singapore

${ }^{2}$ Department of Hepato-pancreato Biliary Surgery, Singapore General Hospital, Singapore

${ }^{3}$ SingHealth Duke-NUS Transplant Centre, Singapore

Correspondence: Prof Bien-Keem Tan, Department of Plastic, Reconstructive and Aesthetic Surgery, Singapore General Hospital, Outram Road, Singapore 169608.

Email: tan.bien.keem@singhealth.com.sg
} 


\section{CLINICAL IMPACT}

\section{What is New}

- The challenges in hepatic artery reconstruction in liver transplantation are related to a mismatch in vessel size between donor and recipient hepatic arteries, and poor quality of recipient vessels.

- A multispecialty approach using modified operating instruments, meticulous microsurgical operating techniques, and interpositional arterial grafts can help maximise the long-term patency of the reconstructed hepatic artery.

\section{Clinical Implications}

- These solutions are particularly applicable to patients in Singapore, whose recipient vessels tend to be smaller and are more affected by previous transarterial interventions or atherosclerosis.

resulting in vessel walls that are fragile (Fig. 1). In this series of 51 patients, we aim to highlight these challenges and discuss our surgical strategies for a successful anastomosis.

\section{METHODS}

A review was conducted of all adult patients who underwent deceased or living donor liver transplantation at the Singapore General Hospital from January 2015 to December 2018. All hepatic artery anastomoses were performed with the aid of an operating microscope by 2 surgeons. Demographic and operative data collected were age, indication for transplantation, type of transplantation, artery calibre and recipient vessel. Outcome data collected were postoperative hepatic artery resistive indices and peak systolic velocities at postoperative days 1, 3, 5, 7, 9 and 14, and any occurrence of biliary leak or hepatic artery thrombosis was recorded. Patients were on a standardised immunosuppression regimen in accordance with the department liver transplant protocol.

\section{Surgical technique}

Following hepatectomy and inset of the donor liver, the liver transplant surgeons proceeded with reconstitution of the hepatic vein and portal vein. This was followed by anastomosis of the hepatic artery by the plastic surgery team, and subsequent biliary reconstruction by the transplant team. The operating microscope, positioned at the patient's left, was used for all hepatic artery anastomosis. Utilising a moist penny towel and a Thompson retractor, the donor liver was retracted superiorly to expose the recipient vessels. A second assistant, standing at the patient's left side, manually retracted any protrusive loops of bowel with a moist penny towel. Vessel preparation and anastomosis were performed at 10x magnification. To obtain a linear arteriotomy, the recipient vessel was supported on an angled cutting platform, and an 11 blade, mounted on an angled blade holder, was used to trim/incise the vessel. The vessel edge was freshened (but not skeletonised) before the arterial wall was coapted in 2 layers. The sutures used for anastomosis were $8 / 0$, and the repair was reinforced with interrupted $9 / 0$ adventitial stitches. Fibrin sealant was applied over the anastomosis to reinforce the watertight seal and also to fix the lie of the vessel. Intraoperative duplex ultrasound was used to confirm patency of the anastomosis.

\section{RESULTS}

There were 51 patients who underwent liver transplantation (Table 1). The mean (range) age was 58 (41-71) years and $69 \%$ of the patients were men $(n=35)$. The most common indications were chronic hepatitis, non-alcoholic steatohepatitis and primary biliary cirrhosis.

There were 31 patients who received deceased donor liver grafts (DDLT) and 20 received living donor liver grafts (LDLT). The mean DDLT graft weight was $1,353.3 \mathrm{~g}$, while the mean LDLT graft weight was $570.5 \mathrm{~g}$. The most common recipient artery was the left or right hepatic artery $(n=31)$, followed by the common hepatic artery $(n=10)$ and gastroduodenal artery $(n=4)$. Dual anastomoses were performed in 5 cases and radial artery interpositional grafts utilised in 4 cases. An end-to-end anastomosis was performed by default; an end-to-side technique was employed in 2 cases due to calibre mismatch. The average time for a single arterial anastomosis was $75 \mathrm{~min}$. The mean (range) postoperative resistive index and hepatic artery peak systolic velocity were $0.68 \mathrm{~m} / \mathrm{s}(0.68-0.79)$ and $0.98 \mathrm{~m} / \mathrm{s}(0.86-1.06)$. One patient developed hepatic artery thrombosis on postoperative day 5. This was detected on ultrasound surveillance, confirmed on computed tomographic angiography, and successfully salvaged with arterial thrombectomy and the use of a radial artery interpositional graft. During the study period, 3 patients died from unrelated causes (sepsis: $\mathrm{n}=2,9$ months and 22 months post-transplant; recurrent hepatocellular carcinoma: $\mathrm{n}=1,7$ months post-transplant). Two patients developed postoperative biliary stenosis that was successfully treated with biliary stenting. In both cases, ultrasound sonography showed 
Table 1. Patient demographics

\begin{tabular}{lc}
\hline Characteristics & N=51 \\
\hline Sex & 16 \\
\hline Female & 35 \\
\hline Male & \\
\hline Age & $58(41-71)$ \\
\hline Age, mean (range), years & \\
\hline Diagnosis & 23 \\
\hline Hepatitis B or C & 11 \\
\hline Non-alcoholic steatohepatitis & 5 \\
\hline Primary biliary cirrhosis & 4 \\
\hline Hepatocellular carcinoma & 3 \\
\hline Alcoholic liver disease & 5 \\
\hline Others & \\
\hline Transplant type & 18 \\
\hline Living donor grafts & 31 \\
\hline Deceased donor liver grafts & \\
\hline Graft weight, mean, $g$ & 570 \\
\hline Living donor grafts & 1353 \\
\hline Deceased donor liver grafts & \\
\hline
\end{tabular}

normal resistive indices and triphasic intrahepatic arterial flow, indicating patency of the hepatic artery anastomosis (Table 2).

\section{DISCUSSION}

In this series of 51 patients, we draw special attention to the microsurgical challenges of hepatic artery reconstruction in liver transplantation. The problems related mainly to vessel adequacy (length, calibre and thickness mismatch) and vessel quality (vessel fibrosis and a preponderance for intimal separation).

\section{Problem 1: Calibre and thickness mismatch}

Some degree of vessel mismatch is always anticipated as patients with liver cirrhosis develop hypertrophy of the coeliac axis from chronic portal hypertension. The difficulties of working with a short vessel stump, particularly with LDLT, are compounded by the deep cavity wherein the recipient vessels reside.

The ideal recipient artery should have adequate length, minimal fibrosis and a good pressure head. The left or right hepatic artery is our first choice. Arising from the bifurcation of the proper hepatic artery at or just
Table 2. Outcome data recorded

\begin{tabular}{|c|c|}
\hline Complications & No. of patients \\
\hline Hepatic artery thrombosis & $1^{\mathrm{a}}$ \\
\hline Biliary stenosis & $2^{b}$ \\
\hline \multicolumn{2}{|l|}{ Mortality } \\
\hline Sepsis & $2^{\mathrm{c}}$ \\
\hline Recurrent hepatocellular carcinoma & $1^{\mathrm{d}}$ \\
\hline Postoperative day number & $\begin{array}{l}\text { Peak systolic velocity, } \\
\text { mean (range), m/s }\end{array}$ \\
\hline 1 & $1.06(0.15-3.12)$ \\
\hline 3 & $0.86(0.20-3.05)$ \\
\hline 5 & $0.95(0.28-2.36)$ \\
\hline 9 & $1.00(0.23-2.13)$ \\
\hline 14 & $1.06(0.15-3.12)$ \\
\hline Postoperative day number & $\begin{array}{l}\text { Resistive index, } \\
\text { mean (range) }\end{array}$ \\
\hline 1 & $0.68(0.33-0.97)$ \\
\hline 3 & $0.69(0.30-0.97)$ \\
\hline 5 & $0.69(0.41-0.84)$ \\
\hline 9 & $0.69(0.40-0.82)$ \\
\hline 14 & $0.69(0.45-0.88)$ \\
\hline
\end{tabular}

${ }^{a}$ postoperative day 5 , salvaged

${ }^{\mathrm{b}}$ hepatic artery Doppler ultrasound normal

c 9 months and 22 months post-transplant

d 7 months post-transplant

proximal to the porta hepatis, the mean diameter of these vessels in our study population was $2.3 \mathrm{~mm}$, making them a good size match for donor vessels up to the level of the proper hepatic artery. For larger calibre donor vessels, the bifurcation of the left and right hepatic arteries at the proper hepatic artery, or the take-off of the splenic artery can be used.

If the hepatic arteries are fibrotic or delaminated, the coeliac axis offers several alternatives. The common hepatic artery, arising from the coeliac axis, runs superolaterally along the upper border of the pancreas to the left side of the portal vein, before splitting into the gastroduodenal artery and the proper hepatic artery. With a mean diameter of $4.2 \mathrm{~mm}$, the common hepatic artery is useful if the donor pedicle is large, with some redundancy. In our series, it was used in DDLT $(n=10)$, where the donor graft was procured at the level of the coeliac trunk or common hepatic artery, or in conjunction with the gastroduodenal artery as part of a dual anastomosis $(n=3)$. 
The gastroduodenal artery is a useful option in patients who have undergone transarterial chemoembolisation as these vessels are spared during treatment. It can also be used in conjunction with the common hepatic artery in a dual anastomosis configuration $(\mathrm{n}=3)$ for large grafts. A branch of the common hepatic artery, it passes inferiorly towards the first part of the duodenum, and bifurcates into the right gastroepiploic and superior pancreaticoduodenal artery. The mean diameter of the gastroduodenal artery was $3.2 \mathrm{~mm}$ in our series. If a larger calibre is required, the bifurcation of the gastroduodenal artery at the common hepatic artery is the preferred location for the anastomosis. The vessel should be prepared carefully as aggressive dissection can cause injury or devascularisation of the pancreatic head.

Other recipient vessels that have been employed included the right gastroepiploic artery ${ }^{3}$ and the distal abdominal aorta (in conjunction with a donor iliac artery vascular graft). ${ }^{4}$ If the donor stump is small but of sufficient length, end-to-side anastomosis can be performed to overcome calibre mismatch. A slit arteriotomy is used, in contrast with a traditional excision type arteriotomy, as it is mechanically advantageous ${ }^{5}$ and minimises vessel manipulation.

\section{Problem 2: Short vessel stump}

In the situation where vessel size and length are inadequate, a radial artery interpositional graft is used as a vascular funnel. With a mean diameter of $4 \mathrm{~mm}$, it serves as a bridge between donor recipient vessels that are severely mismatched (Fig. 2) while allowing for a tension-free anastomosis. Preoperatively, previous percutaneous vascular interventions (intra-arterial blood pressure monitoring, access for coronary angioplasty) should be noted and Allen's test performed.

The radial artery is palpated or traced using Doppler scanning along its axis, just deep to the flexor carpi radialis (FCR). Under tourniquet control, a $5 \mathrm{~cm}$ incision is made on the radial forearm. The FCR is retracted radially to expose the radial artery and a $5-8 \mathrm{~cm}$ segment is harvested. The graft is clamped proximally and flushed through with heparinised saline to check for leaks before the distal anastomosis, at the donor graft, is performed on the back table. In our series, radial artery interpositional grafts were used in 4 cases of LDLT in an end-to-end configuration. In 3 patients, artery grafting was performed primarily to overcome a short donor stump, while in 1 patient who underwent previous TACE, it was performed as a secondary procedure following the development of hepatic artery thrombosis on postoperative day 5 .
The use of saphenous ${ }^{6}$ and common iliac vein grafts ${ }^{7}$ have been reported, but they have been associated with thrombosis rates of up to $23.8 \% .{ }^{8}$ Moreover, in our estimation, arterial grafts were a better match for the hepatic artery as vein grafts were less suited for the transmission of high arterial pressures.

\section{Problem 3: Poor recipient vessel quality}

Due to previous transarterial chemoembolisation, peritonitis or atherosclerosis, the recipient vessels of patients undergoing liver transplantation are fragile, with a tendency to tear or delaminate with manipulation.

To preserve vessel integrity, handling of the adventitia and vessel skeletonising should be minimised during preparation. This process is greatly facilitated with the use of angled instruments (Fig. 3). The vessel is positioned on a cutting platform that is fashioned from a 60-degree angulated steel blade. The microsurgical assistant steadies this buttress while the primary surgeon uses an 11 blade, mounted at a similar angle, to incise the vessel edge in a guillotine fashion. Unlike reusable instruments, the edge of the disposable blade is guaranteed, producing a crisp, uniform vessel edge in a single pass, with minimal disruption of the intima.

\section{Anastomotic techniques specific to hepatic artery reconstruction}

Anastomotic leak is poorly tolerated as the underlying coagulopathy and high driving pressures across the anastomosis may herald postoperative haematoma. We have modified our suture technique to minimise anastomotic leak.

\section{Far and near stitching}

A staggered stitching technique was employed in vessels that have previously undergone chemoembolisation. Due to excessive friability, a linear line of perforations in the vessel wall has a tendency to fissure (Fig. 4). To circumvent this, interrupted stitches were taken at varying distances from the vessel edge to produce a staggered suture line.

\section{Intimal tagging}

In cases where intimal dissection had occurred and further cutback of the vessel was not feasible, the intima was tagged down to the tunica media by passing the suture intraluminally to extraluminally, past the point of separation. If dissection had occurred in both donor and recipient vessels, a double arm suture was used, and the suture introduced intraluminally on both sides, directed past the area of delamination and thence through the vessel wall (Fig. 5). 


\section{Adventitial stitches}

Following completion of the anastomosis with $8 / 0$ or $9 / 0$ suture, reinforcing $9 / 0$ or $10 / 0$ adventitial stitches were applied before the anastomosis was secured with application of fibrin sealant. These external stitches sealed any gaps which may only become evident following release of the vessel clamps.

\section{Case examples}

We discuss 4 case examples from our series.

\section{Case 1: Living donor liver transplant}

\section{Problem: Short stump}

\section{Solution: Radial artery graft}

A 71-year-old man with multifocal hepatocellular carcinoma (HCC) secondary to chronic hepatitis B and a model for end-stage liver disease (MELD) score of 20 underwent LDLT. The graft weight was $661 \mathrm{~g}$. The stump of the donor hepatic artery was $6 \mathrm{~mm}$, necessitating the use of a radial artery interposition graft in order for end-to-end anastomosis to the recipient common hepatic artery. The donor anastomosis was performed on a benchtop before the radial artery was anastomosed to the recipient common hepatic artery. Postoperatively, he developed a biliary anastomotic leak that was managed successfully with endoscopic stenting. Surveillance ultrasound confirmed patent arterial flow
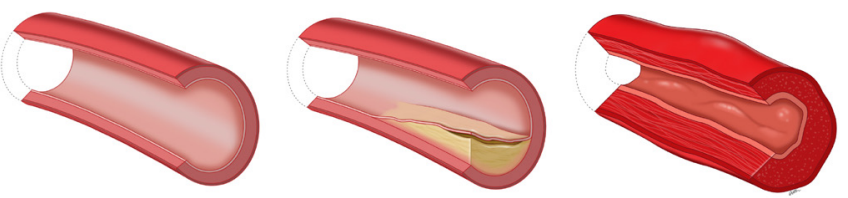

Fig. 1. Pathological condition of atherosclerotic and chemoembolised vessels. Centre: The presence of intimal plaque in atherosclerotic vessels results in a tendency for intimal separation. Right: Previous chemoembolisation renders vessels fibrotic with pan-vessel oedema and fragility.

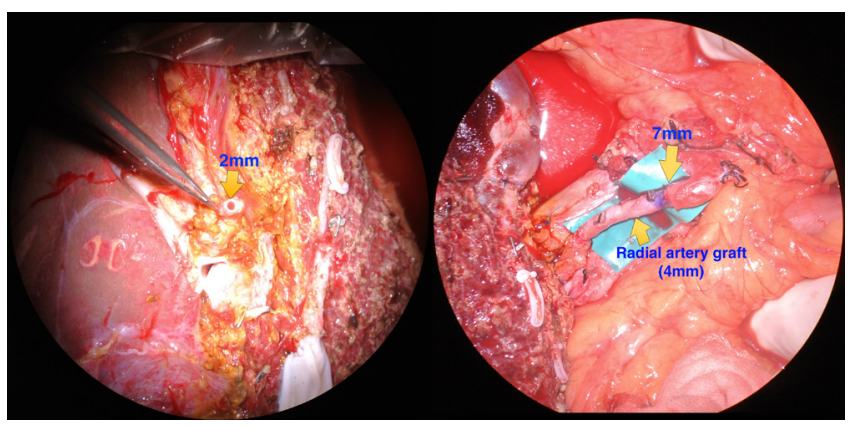

Fig. 2. The radial artery graft ( $4 \mathrm{~mm}$ mean diameter) acts as a vascular funnel in cases of severe mismatch, while allowing for a tension-free anastomosis. with normal resistive indices. He was discharged well 6 weeks postoperatively.

\section{Case 2: Living donor liver transplant}

\section{Problem: Vessel mismatch}

Solution: End-to-side anastomosis

A 63-year-old man with non-alcoholic steatohepatitisinduced cirrhosis and a MELD score of 18 underwent LDLT. A 3:1 size mismatch between the recipient left hepatic artery and donor common hepatic artery branch was noted, necessitating the use of end-to-side anastomosis technique. Postoperative duplex ultrasound showed patent inflow and normal resistive indices. He was discharged well on day 19 postoperatively (Fig. 6).

\section{Case 3: Living donor liver transplant}

Problem: Previous TACE with poor vessel quality; hepatic artery thrombosis

\section{Solution: Salvage with radial artery graft}

A 56-year-old man with recurrent HCC despite 4 previous sessions of transarterial chemoembolisation and radio frequency ablation underwent LDLT. A 2:1 mismatch in vessel size was overcome with standard microsurgical technique, and end-to-end anastomosis between the recipient left hepatic artery and donor right hepatic artery was performed. Initial postoperative

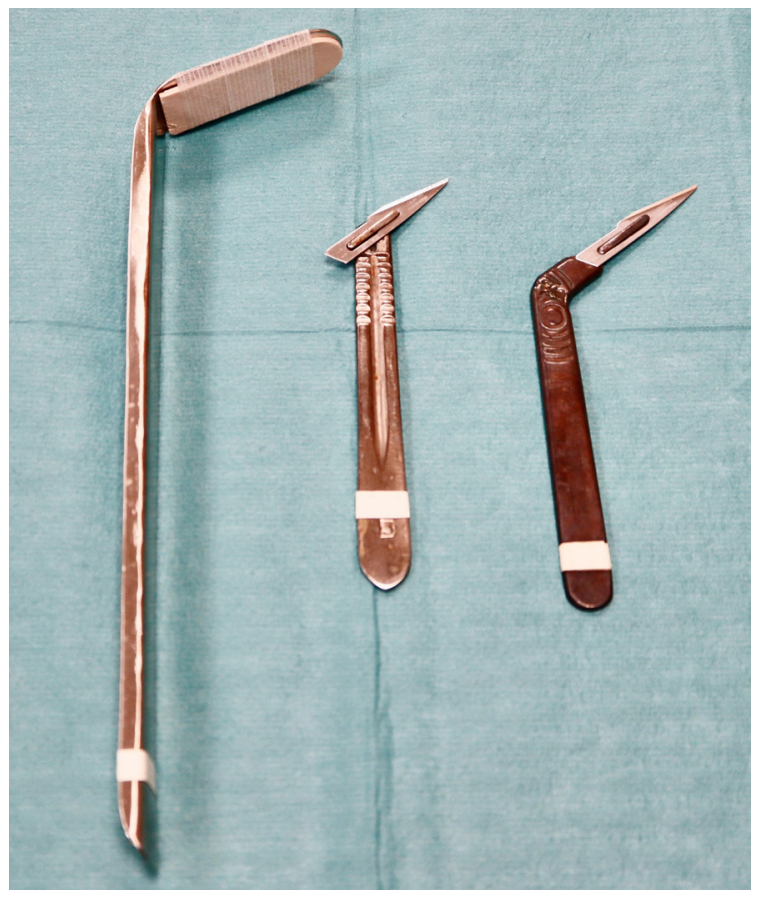

Fig. 3. Angled cutting platform. Angled instruments facilitate vessel preparation and the use of a disposable 11 blade guarantees a sharp cutting edge. 


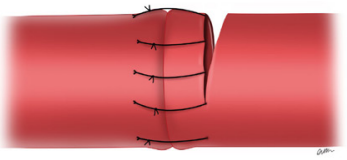

Linear suture line with increased risk for fissure

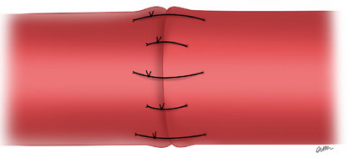

Staggered suture line reduces risk of fissure

Fig. 4. Far-and-near stitching. Left: A linear line of sutures has a tendency to cause fissuring. Right: Interrupted stitches can be taken at variable distances from the vessel edge to create a staggered suture line.
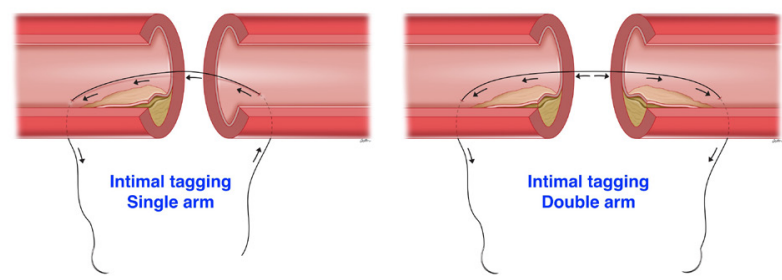

Fig. 5. Intimal tagging sutures. Left: The suture is taken past the point of delamination to tag it down to the tunica media. Right: If both recipient and donor vessels have delaminated, a double arm suture is used.
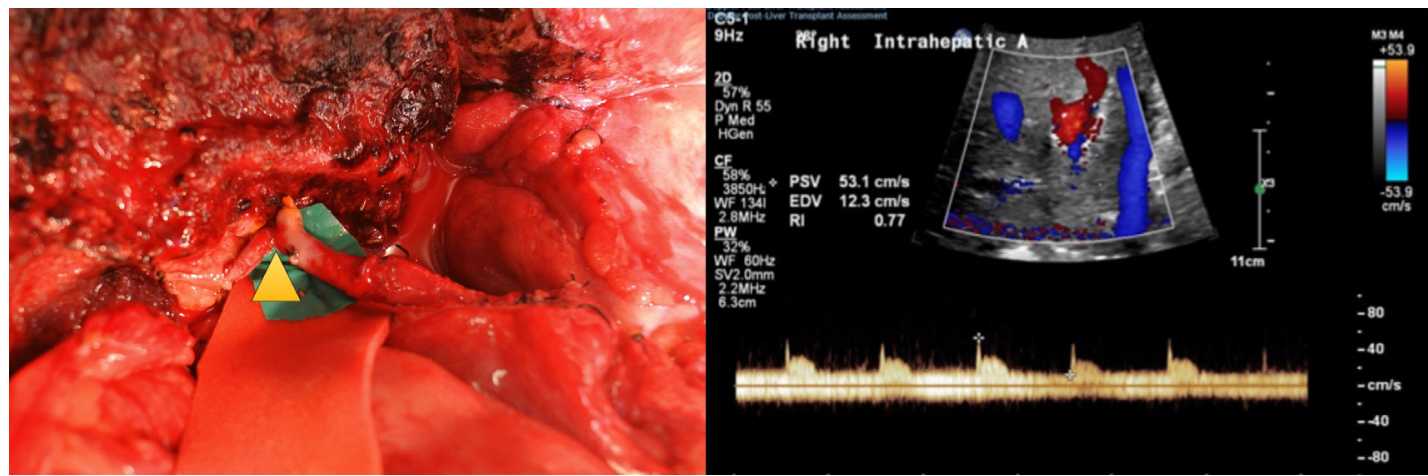

Fig. 6. Living donor liver transplant. Intraoperative photograph showing how a 3:1 vessel calibre mismatch between the recipient left hepatic artery and donor common hepatic artery was overcome. Left: An end-to-side anastomosis was performed. Right: Postoperative ultrasonography showed good flow with a resistive index of 0.77 .

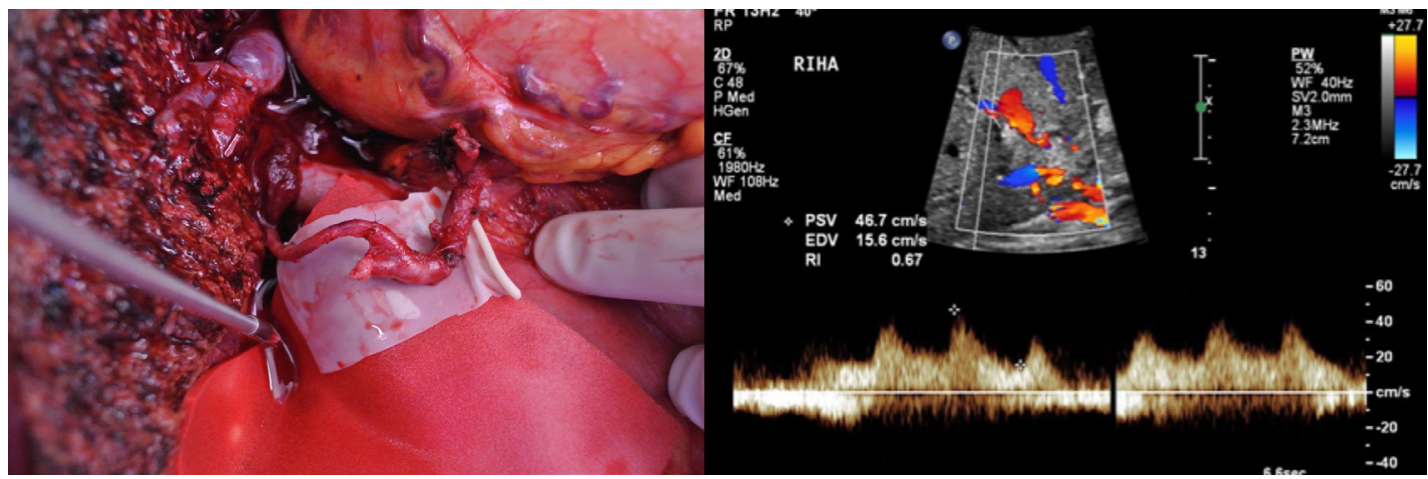

Fig. 7. Living donor liver transplant in a patient with previous transarterial chemoembolisation and radiofrequency ablation. Left: Anastomotic thrombosis was salvaged with an interpositional radial artery graft on postoperative day 5 . Right: Post-salvage ultrasonography showed good flow with a resistive index of 0.67 .

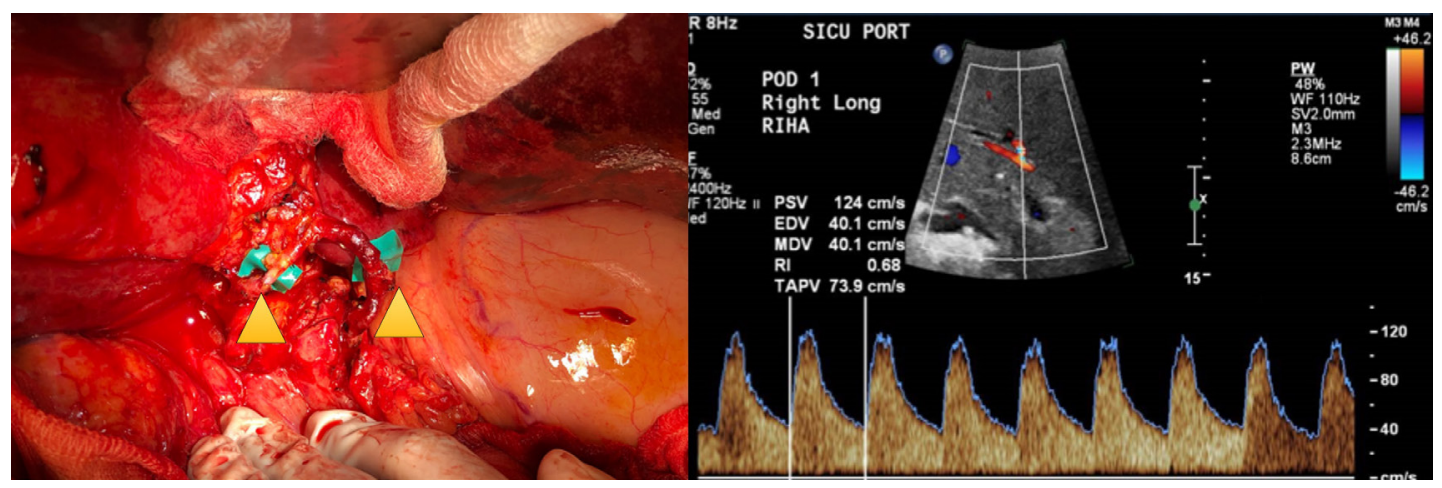

Fig. 8. Deceased donor liver transplant using a large donor liver $(1,500 \mathrm{~g})$. Two separate inflows were created to improve global perfusion. Left: The main inflow was through the common hepatic artery (right arrow), and a second inflow created through the gastroduodenal artery (left arrow) supercharged the graft. Right: The postoperative ultrasonography showed good flow with a resistive index of 0.68 . 
Doppler ultrasound showed a downtrending resistive index of $0.62-0.53$. On postoperative day 5 , the hepatic arteries were not identifiable on Doppler ultrasound. A computed tomography (CT) angiogram confirmed complete thrombosis of the hepatic artery. On investigation, it was found that the posterior vessel wall had delaminated during placement of the posterior stitch. Following unsuccessful balloon thrombectomy, the decision was made to use the gastroduodenal artery with a radial artery interposition graft. CT angiography on day 16 post-revision confirmed a patent anastomosis with enhancement of small calibre intrahepatic arteries. The patient was transferred to a rehabilitation hospital 1 month postoperatively (Fig. 7).

\section{Case 4: Deceased donor liver transplant}

Problem: Large graft size (>1,000g)

\section{Solution: Dual anastomosis}

A 60-year-old woman with acute on chronic hepatitis B flare and fulminant liver failure underwent DDLT. The donor graft was $1,583 \mathrm{~g}$. In view of the significant size of the graft, dual end-to-end anastomosis between the right hepatic artery to the recipient gastroduodenal artery, and left hepatic artery to the recipient common hepatic artery was performed. Postoperative Doppler ultrasound showed patent intrahepatic arterial flow and normal resistive indices. The patient was discharged well on day 21 postoperatively (Fig. 8).

\section{CONCLUSION}

Hepatic artery thrombosis after liver transplantation is poorly tolerated with high morbidity and mortality.
The challenges of hepatic artery reconstruction in liver transplantation are related to vessel quality and adequacy. Recipient vessel selection, radial artery interpositional grafts, selective vessel manipulation and variations in anastomosis technique are useful strategies to maximise the chances of success.

\section{REFERENCES}

1. Northover J, Terblanche J. Bile duct blood supply. Its importance in human liver transplantation. Transplantation 1978;26:67-9.

2. Imagawa DK, Busuttil RW. Technical problems: vascular. In: Busuttil RW, Klintmalm GB. Transplantation of the liver, 2nd ed. Pennsylvania: WB Saunders; 2005.

3. Ikegami T, Kawasaki S, Hashikura Y, et al. An alternative method of arterial reconstruction after hepatic arterial thrombosis following living-related liver transplantation. Transplantation 2000; 69:1953-5.

4. Shaw BW Jr, Iwatsuki S, Starzl TE. Alternative methods of arterialization of the hepatic graft. Surg Gynecol Obstet 1984; 159:490-3.

5. Tan BK, Wong CH, Chew W, Hong SW. Use of the slit arteriotomy for end-to-side arterial anastomosis in free-tissue transfers to the extremities. J Plast Reconstr Aesthet Surg 2009;62:1519-23.

6. Garcia-Valdecasas JC, Grande L, Rimola A, et al. The use of the saphenous vein for arterial reconstruction in orthotopic liver transplant. Transplant Proc 1990;22:2376-7.

7. Asakura T, Ohkohchi N, Orii T, Koyamada N, et al. Arterial reconstruction using vein graft from the common iliac artery after hepatic artery thrombosis in living-related liver transplantation. Transplant Proc 2000;32:2250-1.

8. Tzakis AG, Gordon RD, Shaw BW Jr, et al. Clinical presentation of hepatic artery thrombosis after liver transplantation in the cyclosporin era. Transplantation 1985;40:667-71. 